\title{
Secondary syphilis: Report of a case complicated by atypical Jarisch-Herxheimer reaction
}

DAVID R. WOOD, MAJ, MC, USA

San Antonio, Texas
Syphilis is a multi-system disease characterized by several welldefined clinical stages. In the absence of therapy, those stages may progress from primary to secondary syphilis, and finally to early then late latent syphilis. The patient described presented with concomitant secondary syphilis and Schamberg's dermatosis. Penicillin G benzathine was administered intramuscularly, and was followed in 3 days by an inability to void, impotence, constipation with bloody stools, low back pain, and paresthesias of the perineum. Spinal tap revealed neurosyphilis. Intravenous penicillin $G$ (2-3 million units every 4 hours) was administered for 15 days. Except for the Schamberg's dermatosis, all of the clinical findings resolved over 1-5 weeks. This case is explained best as secondary syphilis complicated by an atypical Jarisch-Herxheimer reaction "therapeutic shock" with asymptomatic neurosyphilis.

The multisystemic disease syphilis is characterized by several well-defined clinical stages. ${ }^{1-5}$ Syphilis obviously is not rare, but even with a classic presentation, the disease may be difficult to recognize. This paper describes the unusual presentation of a case of secondary syphilis and the events subsequent to therapy, which were attributed to "therapeutic shock."

\section{Report of case}

A 49-year-old white man presented to Brooke Army Med- ical Center on December 22, 1984, for evaluation of a rash, which had begun on the right leg in September 1984. This was associated with the onset of annular erythematous macules with central clearing on the genitals in November 1984. The patient had a history of gonorrhea treated with penicillin in 1958. There was no history of chancre. A routine Venereal Disease Research Laboratory (VDRL) test in 1980 and a rapid plasma reagin (RPR) test in 1983 were nonreactive. Finally, the patient denied homosexual activity, but described a sexual contact in October 1984 as someone who might have the disease.

Biopsy of the leg was performed in January 1985, with findings consistent with Schamberg's disease. Schamberg's disease is a progressive pigmentary dermatosis which is most common in middle-aged men and presents in the lower legs. The biopsy site was complicated by cellulitis, and the patient was treated with oral erythromycin ( $500 \mathrm{mg}$. every 6 hours for 5 days). On February 21, an RPR test was reported to be positive in a titer of 1:256, with a fluorescent treponemal antibody titer of 1:128. The patient refused lumbar puncture. Penicillin $\mathrm{G}$ benzathine was administered intramuscularly in a dose of 2.4 million units on March 7,14, and 20. On March 10, the patient reported an increasing inability to void spontaneously, impotence, increasing constipation with bloody painful stools, low-back pain, and paresthesias of the perineum. Dysuria was not present. A urinary catheter was inserted on March 21, and cystometrography performed on March 26 revealed a large, flaccid bladder without obstruction. The patient was hospitalized on March 26.

Physical examination revealed no oral, anal, or penile lesions. The patient had a lax anal sphincter, and the stool tested positive for blood. Reddish-purple macules were present on the legs. Inguinal lymphadenopathy was palpated. Neurologic examination revealed normal strength, vibratory sense, proprioception, light touch, muscle stretch reflexes, cerebellar function, and cranial nerves. The erythrocyte sedimentation rate was $60 \mathrm{~mm}$./ $\mathrm{hr}$. On lumbar puncture, the cerebrospinal fluid was clear 
and colorless, with values as follows: white blood cells, 94/cu.mm. (92 percent lymphocytes); red blood cells, 9/ cu.mm.; protein, $119 \mathrm{mg}$./dl. (normal, $\leqslant 45 \mathrm{mg} . / \mathrm{dl}$.), glucose, $64 \mathrm{mg} . / \mathrm{dl}$. (serum value, $101 \mathrm{mg} . / \mathrm{dl}$.), and VDRL titer, 1:4. Intravenous crystalline penicillin G (3.3 million units every 4 hours) was initiated for 15 days on March 30.

Proctoscopy on March 29 and air-contrast barium enema on April 4 had normal results. The indwelling urinary catheter was discontinued on April 1, by which time the hemocult positive stools and constipation also had been completely resolved. The low-back pain and perineal paresthesias were resolved by April 7, and the impotence by May.

On June 24, lumbar puncture revealed a clear, colorless fluid, with the following values: white blood cells, 8 / cu.mm. (79 percent lymphocytes); red blood cells, 2/ cu.mm.; protein, $52 \mathrm{mg} . / \mathrm{dl}$.; glucose, $53 \mathrm{mg} . / \mathrm{dl}$.; and VDRL titer, 1:1. The patient remained asymptomatic on routine follow up in June 1985, January 1986, and September 1986. Schamberg's dermatosis remains on the legs.

\section{Discussion}

Syphilis has a myriad of possible signs and symptoms, which may lead to some confusion in diagnosis, as occurred in the present case. Mucocutaneous lesions are present in 80 percent of patients with secondary syphilis. ${ }^{2}$ It is classically described as bilaterally symmetric, with involvement of the trunk, proximal extremities, and the palms and soles, ${ }^{1,4,5}$ and it may imitate many other dermatologic disorders. ${ }^{5}$ Untreated, the skin lesions of secondary syphilis tend to resolve over 4 to 12 weeks. ${ }^{1}$ In the present case, the genital rash was a manifestation of secondary syphilis, while the rash on the legs was diagnosed correctly as Schamberg's dermatosis.

Asymptomatic cerebrospinal fluid abnormalities occur in 25-30 percent of persons with secondary syphilis, and symptomatic meningitis occurs in 1-2 percent. $1,2,4,6$ These include pleocytosis, elevated protein value, and a positive VDRL reaction. The VDRL test is recognized as being very specific but not sensitive for the diagnosis of neurosyphilis. ${ }^{7,8}$ Neuropathy of the cranial nerves, especially II, III, VII, and VIII, is common ${ }^{1,2,4,6}$; the mechanism appears to be obliterative small-vessel endarteritis. ${ }^{4}$ Treatment of secondary syphilis usually results in resolution of associated neuropathy without exacerbation; however, persistent deficits have been reported. ${ }^{2}$

Neuropathy of the sacral plexus is unlikely. Tabes dorsalis is characterized by chronic inflammation of the spinal cord and spinal roots, especially the lumbar and sacral dorsal roots. ${ }^{3-5,9}$ This disorder produces difficulty with urination, impotence, absent muscle stretch reflexes, loss of deep pain response, prominent paresthesias, and loss of proprioception. The urinary bladder involvement may be irreversible in tabes dorsalis. ${ }^{10}$

In the late stage of cerebrospinal syphilis, various syndromes may occur, among them Erb's syphilitic spinal paralysis. ${ }^{10}$ This disorder initially may present with only bladder disturbances and no sensory changes, but it eventually can result in severe pain and a transverse lesion of the spinal cord. Symptomatic neurosyphilis previously was associated with classic findings, but in this postantibiotic era the clinical manifestations and cerebrospinal fluid findings can be variable. ${ }^{3,7}$ With treatment, the neurologic deficits of late syphilis generally stabilize, but they have been reported to irreversibly exacerbate. ${ }^{3}$ Neurologic deficits associated with neurosyphilis have been reported to progress after treatment; this progression was attributed either to damage incurred prior to treatment ${ }^{4}$ or to an autoimmune process. ${ }^{9}$ However, this is questionable, because the use of high-dose ( 20 million units daily) of penicillin results in the immediate arrest of signs and symptoms. ${ }^{11}$

An unusual form of the Jarisch-Herxheimer reaction (JHR) to antibiotic therapy for secondary syphilis may explain what happened to our patient, although he reported none of the constitutional symptoms generally associated with JHR (fever, chills, rash, malaise, headache, myalgias, tachycardia, and hypotension). The exact mechanism of this reaction is unknown, but it is thought to be the result of toxins released with the killing of spirochettes. ${ }^{1,5}$ The JHR is common in secondary syphilis (75-90 percent), ${ }^{2}$ less so in late syphilis (25-30 percent) ${ }^{4}$ and affects only 2 percent of those with neurosyphilis. ${ }^{3}$ The constitutional symptoms generally begin within 4 hours of antibiotic administration and subside within $16-24$ hours. ${ }^{1,5} \mathrm{~A}$ temporary exacerbation of skin and neurologic symptoms may be seen with this reaction. 1,2,4,6

Treatment of secondary syphilis, as recommended by the Centers for Disease Control (CDC) ${ }^{12}$ is intramuscular penicillin $\mathrm{G}$ benzathine in a dose of 2.4 million units administered at a single session. Syphilis of longer than 1 year's duration or of indeterminate duration also should be treated with intramuscular penicillin $\mathrm{G}$ benzathine (2.4 million units weekly for 3 consecutive weeks, to a total of 7.2 million units). ${ }^{12}$ The $\mathrm{CDC}^{12}$ regimen for neurosyphilis is aqueous crystalline penicillin $\mathrm{G}$ (2-4 million units administered intravenously every 4 hours, to a total of 12-24 million units daily) for 10 days, followed by intramuscular penicillin $\mathrm{G}$ benzathine ( 2.4 million units weekly) for three doses. An alternative regimen for neurosyphilis offered by the CDC is just 3 consecutive 
weekly doses of intramuscular penicillin $\mathrm{G}$ benzathine (2.4 million units weekly). This discrepancy in penicillin dosing and delivery recommendations represents the controversy surrounding this subject. Data from Mohr and associates ${ }^{11}$ and Tramont ${ }^{13}$ would support the use of high-dose ( 20 million units daily) intravenous aqueous crystalline penicillin $\mathrm{G}$ over a 10 -day period.

\section{Comment}

The sequence of events surrounding the patient in this case can best be explained as secondary syphilis complicated by atypical JHR with asymptomatic neurosyphilis. Proctitis has been reported to occur in secondary syphilis ${ }^{2}$ and is a likely cause of the painful, bloody stools. Reports by Mohr and associates ${ }^{11}$ and Tramont ${ }^{13}$ would suggest that the initial penicillin therapy for this patient was inadequate to resolve the neurosyphilis. The study by Mohr and associates ${ }^{11}$ revealed no detectable cerebrospinal fluid penicillin in 12 of 13 patients given intramuscular penicillin $\mathrm{G}$ benzathine in a dose of 3.6 million units. It has been established that the minimum acceptable penicillin level for the cerebrospinal fluid is $0.03 \mathrm{mg}$./ ml. $2,3,11$

Neuropathy of the sacral plexus has not been reported. The reported neuropathies involving the cranial nerves of secondary syphilis do not exacerbate with therapy, which reduces the likelihood of that diagnosis. Symptomatic neurosyphilis is unlikely in our patient because of the complete resolution of the signs and symptoms. The sign and symptom complex is not consistent with cerebrospinal syphilis, tabes dorsalis, or progressive paresis. The manifestations of neurosyphilis may begin 6 months to 30 years after exposure, but they generally do not onset for 2-5 years. ${ }^{2,3,10}$ The nonreactive VDRL in 1980 and RPR in 1983 makes a diagnosis of late syphilis with relapse unlikely. Finally, the patient had a questionable sexual contact in October 1984. Therefore, secondary syphilis with asymptomatic neurosyphilis complicated by an atypical JHR "therapeutic shock" 5 appears to be the best diagnosis for this case.

The views expressed in this paper are those of the author and do not necessarily reflect the views of the US Army or the Department of Defense.

1. Drusin, L.M.: Syphilis. Clinical manifestations, diagnosis, and treat ment. Urol Clin North Am 11:121-30, Feb 84

2. McPhee, S.J.: Secondary syphilis. Uncommon manifestations of a common disease. West J Med 140:35-42, Jan 84

3. Hotson, J.R.: Modern neurosyphilis. A partially treated chronic meningitis. West J Med 135:191-200, Sep 81

4. Holmes, K.K.: Syphilis. In Harrison's Principles of Internal Medicine, edited by K.J. Isselbacher. Ed. 9. McGraw-Hill Book Company, 1980, pp. 716-26

5. U.S. Department of Health, Education, and Welfare: Syphilis. A synopsis. U.S. Government Printing Office, Washington, D.C., 1967, pp. 44, 57-69, 76-81, 118-119

6. Verduijn, P.G., and Blecker, J.D.: Secondary syphilis of the facial nerve. Arch Otolaryngol 108:382-4, Jun 82

7. Lee, J.B., et al.: Symptomatic neurosyphilis. Int J Dermatol 22:577-80, Dec 83

8. Dans, P.E., et al.: Inappropriate use of the cerebrospinal fluid Venereal Disease Research Laboratory (VDRL) test to exclude neurosyphilis. Ann Intern Med 104:86-9, Jan 86

9. Jablecki, K.: Infections. In Neurology for non-neurologists, edited by W.C. Wiederholt. Academic Press, New York, 1982, pp. 318-9

10. Mumenthaler, M.: Syphilis of the central nervous system. In Neurology and neurosurgery 1977. Year Book Medical Publishers, Chicago, 1977, pp. 214-9

11. Mohr, J.A., et al.: Neurosyphilis and penicillin levels in cerebrospinal fluid. JAMA 236:2208-9, 8 Nov 76

12. Centers for Disease Control. 1985 STD treatment guidelines (supplement). MMWR 34:94S-6S, 18 Oct 85

13. Tramont, E.C.: Persistence of Treponema pallidum following penicillin G therapy. Report of two cases. JAMA $236: 2206-7,8$ Nov 76

Accepted for publication in July 1986. Updating as necessary, has been done by the author.

Dr. Wood is chief of the Internal Medicine Clinic, Department of the Army, Brooke Army Medical Center, Fort Sam Houston, Texas.

Dr. Wood, 7531 Robin Rest, San Antonio, Texas 78209. 